\title{
Research Article Gravitational Capture of Asteroids by Gas Drag
}

\author{
E. Vieira Neto and O. C. Winter \\ FE-Guaratinguetá, Departamento de Matemática, UNESP-Universidade Estadual Paulista, \\ 12.516-410 SP, Brazil
}

Correspondence should be addressed to E. Vieira Neto, ernesto@feg.unesp.br

Received 30 July 2009; Revised 6 November 2009; Accepted 1 December 2009

Recommended by Silvia Maria Giuliatti Winter

Several irregular satellites of the giant planets were found in the last years. Their orbital configuration suggests that these satellites were asteroids captured by the planets. The restricted three-body problem can explain the dynamics of the capture, but the capture is temporary. It is necessary some kind of dissipative effect to turn the temporary capture into a permanent one. In this work we study an asteroid suffering a gas drag at an extended atmosphere of a planet to turn a temporary capture into a permanent one. In the primordial Solar System, gas envelopes were created around the planet. An asteroid that was gravitationally captured by the planet got its velocity reduced and could been trapped as an irregular satellite. It is well known that, depending on the time scale of the gas envelope, an asteroid will spiral and collide with the planet. So, we simulate the passage of the asteroid in the gas envelope with its density decreasing along the time. Using this approach, we found effective captures, and have a better understanding of the whole process. Finally, we conclude that the origin of the irregular satellites cannot be attributed to the gas drag capture mechanism alone.

Copyright (C) 2009 E. Vieira Neto and O. C. Winter. This is an open access article distributed under the Creative Commons Attribution License, which permits unrestricted use, distribution, and reproduction in any medium, provided the original work is properly cited.

\section{Introduction}

The giant planets of the Solar System have two kinds of satellites, the regular and the irregular ones [1]. This definition was based on the orbits of the satellites. The regular satellites have their orbits near the equatorial plane, the eccentricity is near zero, and they are close to their planets. The irregular ones are out of plane, many are retrograde, the eccentricities are above 0.1 , and they are far from their planets.

Since the discovery of two new Uranus' moons in 1997 by Gladman et al. [2], 98 other satellites were found. These new satellites, plus the old irregulars satellites, are almost all retrograde, as we can see in Table 1.

The orbits of the irregular satellites suggest that they were captured by their planets. That is, they were formed elsewhere in the Solar System like any other asteroid, and later they might have had a close encounter with a planet and could have been captured. 
Table 1: Number of irregular satellites of the giant planets [3].

\begin{tabular}{lccc}
\hline Planet & Prograde & Retrograde & Total \\
\hline Jupiter & 6 & 49 & 55 \\
Saturn & 8 & 27 & 35 \\
Uranus & 1 & 8 & 9 \\
Neptune & 3 & 4 & 7 \\
\hline
\end{tabular}

Using the dynamics of the three-body problem it is possible to explain the gravitational capture of these satellites. But the gravitational capture is temporary (see, for example, [4]). There are works that studied the capture time [5] or the directions of capture [6] in the case of the three-body problem. Thus, it is necessary some kind of additional effect to turn the temporary capture into a permanent one.

In the literature we can find several mechanisms which were proposed in order to turn the capture permanent. It could be a pull-down mechanism due to the mass growth of the planet [7-9], or a capture through n-body interactions [10-12]. In this work we will explore the gas drag mechanism. In the early history of the solar system, in the last stage of the giant planets formation, a gas envelope was formed around each one of them [13]. This gas envelope could make a flying by asteroid to loose enough energy in order to became a prisoner of the planet. Other authors already discussed this possibility (see, for example, [14]). Although the theory and numerical simulation have some success in explaining the prograde satellites, the retrograde ones are not well explained. This is because the retrograde satellites feel a stronger headwind which make them to spiral inward faster and collide with the planet sooner than the prograde ones. This suggests distinct conditions for the gas drag capture of the prograde and retrograde satellites.

Cuk and Burns [14] studied the case of a prograde satellite capture, using the orbital and physical parameters of Himalia Jupiter's satellite. They used a static surface density gas envelope with edges to modify the size of the envelope. They vary the position of the edge, but maintaining the surface density of the gas envelope constant. They have discussed the effect of a gas envelope with decaying density in their work, but made no simulation. Also in the work of Cuk and Burns [14], particles were started inside the gas envelope, mainly using Himalia's initial conditions, and integrated backwards in time.

In the work of Pollack et al. [15] they simulate the formation of a giant planet and characterized the phases: (1) accretion of solid material; (2) accretion of solid material and gas with a small rate; (3) runaway gas accretion. They concluded that the overall time scale is determined by the second phase, and the first and third phase are very quick. They suppose that the envelope did not collapse in the phase of runaway gas accretion as long as the solar nebula could supply the planet with gas rapidly enough. In their work they do not estimate a time interval for the third phase, but their Figure 1(a) give us a clue of this time interval and it seems to last less than a thousand years. In our work we will consider the very last stage, when there is no more gas in the solar nebula to feed the planetary gas envelope and this envelope collapses. We suppose the duration of this stage to be about a hundred years and in this stage the density of the gas envelope will vary until vanish.

In this work we simulate a gas envelope with no edges, but its surface density varies linearly with time. We started the integration of the asteroid orbit outside the gas envelope in a proper region as an heliocentric orbit, and integrated it forward in time until it reaches the gas envelope around the planet. We also point out that this work is in two dimensions, while the work of Cuk and Burns [14] was three-dimensional. 


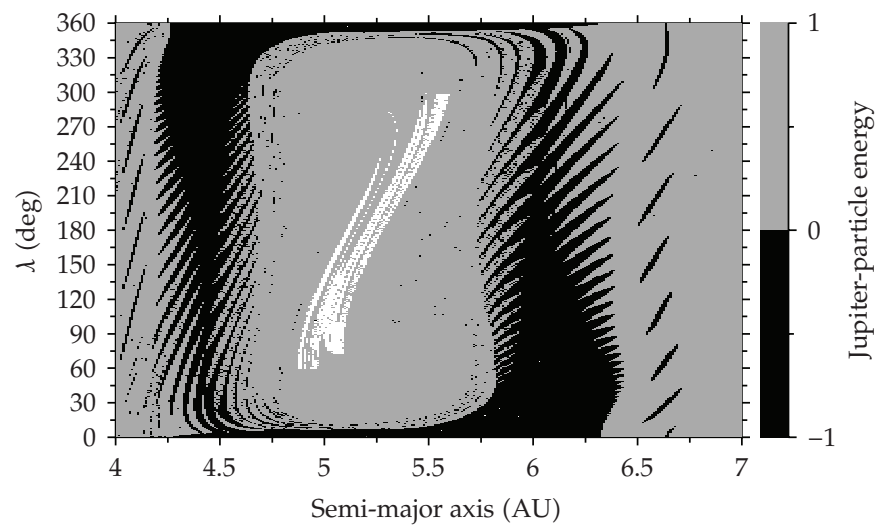

Figure 1: Grid of initial conditions of the asteroid with respect to the Sun. In the plot we can see which initial conditions that lead to negative two-body energy w.r.t. Jupiter, that is, the asteroid was temporarily captured by Jupiter. The white region in the middle of the plot are trajectories which lead to a two-body energy greater than one. For each asteroid initial condition in the $a \times \lambda$ space, Jupiter begins its orbit at 5.2 AU from the Sun with $\lambda=0^{\circ}$.

In our work we will discuss the very last stage of the gas envelope when it collapses onto the planet. Thus, the surface density of the gas will change from some accepted initial value down to zero, vanishing the gas. This configuration is favorable for the retrograde satellites. Our goal is to investigate how to get permanent gravitational capture for the retrograde satellites.

We will first discuss, in Section 2, the possible heliocentric orbital region that could make an asteroid to be captured by Jupiter. In Section 3 we will expose the gas model and show the results of our simulations. Finally, we discuss our results in Section 4.

\section{Possible Origins for the Captured Asteroids}

In order to study the asteroids initial conditions which lead to capture by the planet, we used the circular planar Sun-Jupiter system, and made a grid of initial conditions with semi-major axis and true longitude $(\lambda)$. The others asteroid's initial conditions parameters were fixed in such a way that the asteroid had a circular planar orbit with respect the Sun. The semimajor axis $(a)$ was varied from 4 to 7 Astronomical Units (AU) and the true longitude $(\lambda)$ from $0^{\circ}$ to $360^{\circ}$. For each trajectory in this grid, we measured the two-body energy of the asteroid relative to Jupiter from time $t=0$ to time $t=10^{5}$ years and the minimum value was stored. The two-body energy is not constant in the three-body system and will vary due to the Sun's perturbation. We were interested in the negatives values of the two-body energy, which configures the temporary gravitational capture [16], since negative value of this energy corresponds to an osculate elliptical orbit. Other possible option for this study would be the minimum distance between asteroid and Jupiter and a passage through the Hill sphere, but the energy was chosen because it takes the position and velocity of the particle with respect to the planet, and we can avoid a close passage with very high speed which is not interesting to our experiment.

In the search of proper initial conditions which leads to gravitational capture we did not consider the gas drag. The idea in this first moment is just to find the path for an encounter with Jupiter. 


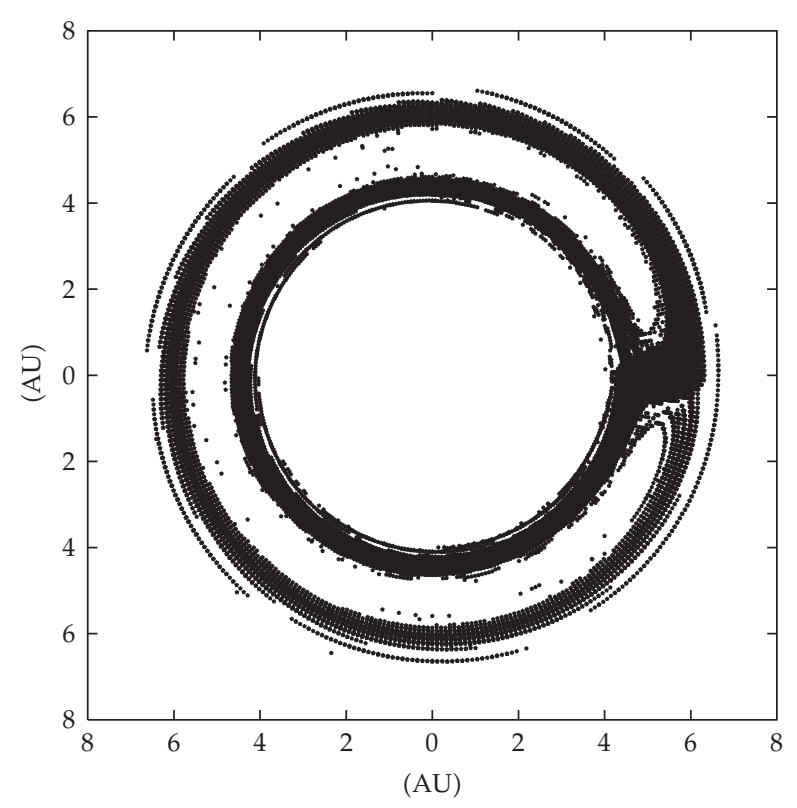

Figure 2: Asteroid initial conditions which lead to negative two-body energy w.r.t. Jupiter, taken from Figure 1, in polar coordinates.

The numerical integrations we made in this work used an integrator that uses GaussRadau spacings described by E. Everhart[17].

The result for this simulation can be seen in Figure 1, where we separated the initial conditions in the grid that lead to positive and negative two-body energy. The black points indicate the initial conditions of trajectories which achieved a minimal negative two-body energy at some point along the integration, while the other color dots correspond to those trajectories whose minimum energy was positive.

On Figure 2 we plotted only the initial conditions which lead to negative two-body energy of the asteroid with respect to Jupiter, in polar coordinates. From these two figures we can see the horseshoe libration zone [18] and the chaotically-unstable region around Jupiter's orbit, which is due to overlap of mean motion resonances [19].

In Figures 1 and 2 we can see the existence of two dense regions with initial conditions that lead to negative values of the two-body energy. These initial conditions are the ones that lead the asteroid to pass close enough to the planet and have a temporary gravitational capture.

\section{Dynamics and Numerical Results}

In order to turn a temporary capture into a permanent one, we used a drag force proportional to the velocity of the asteroid relative to the velocity of the gas, the cross section of a spherical asteroid, and the local density of the gas [20,21]:

$$
\vec{F}_{d}=-C_{d} \frac{\pi}{2} R^{2} \rho(r) v_{\text {rel }} \vec{v}_{\text {rel }},
$$


where $C_{d}$ is the drag coefficient, $R$ is the asteroid radius, $v_{\text {rel }}$ is the relative velocity of the asteroid with respect to the gas, and

$$
\rho(r)=\rho_{0}\left(\frac{r}{r_{0}}\right)^{r}
$$

where $\rho(r)$ is the gas density at a distance $r$ from the centre of the planet, and $\gamma$ is the exponent that gives how the gas density decay with the distance from the planet. In this work we used $r=-1$, as in Cuk and Burns [14]. We also used

$$
\rho_{0}=\frac{\Sigma_{0}}{\sqrt{\pi} H_{0}}
$$

as the gas density at $r_{0}$, with $\Sigma_{0}$ being the gas surface density, and $H_{0}$ the gas envelope height. We considered $r_{0}=100$ Jupiter Radius (JR), $H_{0}=0.05 r_{0}$, as in Cuk and Burns [14]. The asteroidal radius was took as $R=6 \mathrm{~km}$. We choose this small radius because we are interested in the retrograde satellites and this will reduce the decaying effect of the gas. Besides, this is a representative radius value for the retrograde satellites observed around Jupiter. The gas velocity rotation around the planet was chosen to be $90 \%$ of the Keplerian velocity.

In order to change the density of the gas envelope during the passage of the asteroid we implemented the following surface density time function:

$$
\Sigma_{0}(t)= \begin{cases}\Sigma_{0}\left(t_{0}\right), & t<t_{0} \\ \Sigma_{0}\left(t_{0}\right)+\alpha\left(t-t_{0}\right), & t_{0} \leq t \leq t_{1} \\ 0, & t>t_{1}\end{cases}
$$

where $\alpha$ is the time rate of the decreasing density. An exponential (or sigmoid) decay law will change only slightly our results.

The gravitational model used was the general three-body model, that is, the mass of the asteroid were considered. The density of the asteroid was considered $1.5 \mathrm{~g} / \mathrm{cm}^{3}$, and the gas density surface considered was $\Sigma_{0}\left(t_{0}\right)=10^{3} \mathrm{~g} / \mathrm{cm}^{2}$, as in Cuk and Burns [14].

The gas envelope is formed after the consumption of all the gas from the solar nebula. The lack of gas to fuel the planet makes the envelope collapses into the planet $[20,22]$. It is believed that this collapse is quick and took only some hundreds of years to occur. That is, the surface density of the gas goes to zero in times of the order of $10^{2}$ years.

\subsection{Asteroid's Passage through the Gas Envelope}

In order to study the passage of the asteroid through the gas envelope we use the results from Figure 1 to choose an adequate set of initial conditions. The initial conditions used are in the same plane of the gas envelope around Jupiter. We fixed a time interval of 100 years $\left(\Delta t=\left|t_{1}-t_{0}\right|\right)$ for the gas to vanish. The gas has its density changed according to (3.4), from $\Sigma_{0}\left(t_{0}\right)$ to zero in a time interval of $\Delta t=100$ years, and after this period there is no more gas around the planet, $\Sigma_{0}(t)=\Sigma_{0}\left(t_{1}\right)=0$.

The effect of the change of the surface gas density on the asteroid trajectory will be analysed by fixing the initial orbital configuration of the asteroid and Jupiter, but selecting different values of initial integration time from $t=-100$ years to $t=0$ year, with steps of 0.1 
year, while time variation of the gas envelope has ever the initial time $t_{0}=-100$ years and final time $t_{1}=0$ year. That is, for each trajectory the asteroid and Jupiter have the same orbital elements with respect to the Sun, but the orbital integration of the system starts at different times, reaching the gas envelope at different surface density values $\Sigma_{0}$.

During the integration of the trajectory we monitored the relative two-body energy of the asteroid relative to Jupiter to verify its sign. Initially the asteroid is in heliocentric orbit and has a positive relative two-body energy with respect to Jupiter. We stoped the integration if one of the following happens: (i) the asteroid passes through the gas envelope; (ii) the asteroid collides with Jupiter; (iii) the integration time surpass $t=50$ years.

In the first case, to know that the asteroid had passed through the gas envelope around Jupiter, we measured the relative two-body energy of the asteroid with respect to Jupiter during the encounter. The two-body energy turn into negative when the asteroid is close to Jupiter. Then, the relative two-body energy turn to positive again when the asteroid is distant from the planet.

For the second case, as we do not consider the Galilean satellites in the experiment, the collision with Jupiter is considered when the distance Jupiter-asteroid is smaller than the Jupiter's radius.

The third case happens if one of the following occurs: (a) the asteroid is permanently captured; (b) the asteroid is deflected by the gas envelope; (c) the asteroid is temporarily captured.

Case (a) is considered when the asteroid entered in the gas envelope, that is, its relative two-body energy was turned into negative, in a time that the surface density of the gas has a value between $\Sigma_{0}\left(t_{0}\right)$ e 0 . Due to the gas, the asteroid suffers a drag and loose energy. But the gas surface density vanishes, the asteroid stop to loose its energy and, if it lost enough energy, it became a permanent satellite.

In case (b) the asteroid never reach a negative relative two-body energy with respect to Jupiter. In these kind of trajectories the asteroid passes in the outer layers of the gas envelope and it is deflected.

Finally, in case (c) the asteroid arrives at the planet when the surface density of the gas is already zero, or very low, and do not loose enough energy to be effectively captured.

We tested four initial conditions for semi-major axis at 6.0 AU, and other four initial conditions for semi-major axis at $4.5 \mathrm{AU}$. Each one with a different true longitude value $(\lambda)$ for the asteroid. These initial conditions were obtained from Figure 1. In Figure 3 we can see the results of the trajectories of the asteroid which have initial semi-major axis of $6.0 \mathrm{AU}$. None of the trajectories was effectively captured by the planet, many trajectories entered the gas envelope and escaped quickly. Other trajectories never entered the gas envelope as shown in Figures 3(c) and 3(d), where there is a region in the interval $[-100,-40]$ years with no points. This occurs because the trajectories are deflected by the gas. In Figure 3(d), the lack of points for the region $[-7,0]$ happens because the trajectories are with negative two-body energy, but the gas has low surface density and the trajectories will escape again due to the fact that the dissipative effect is too weak.

In Figure 4 we show the results for other four initial conditions, but now for $a=$ 4.5 AU. The plots in the Figure show the time spent by the asteroid inside the gas envelope with negative two-body energy. Only for Figure 4(c) effective captures occur, as showed by the blue squares. In the other three cases there are only collisions and passages near the planet.

We also note in the plots the existence of upward spikes. These spikes occur due to the trajectories transition between trajectories which just pass through the gas envelope and 


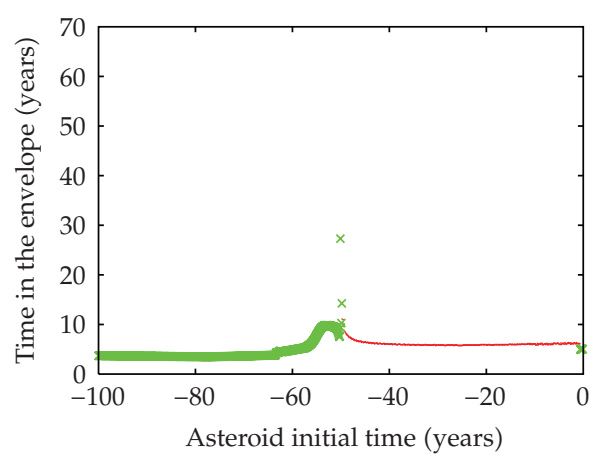

(a)

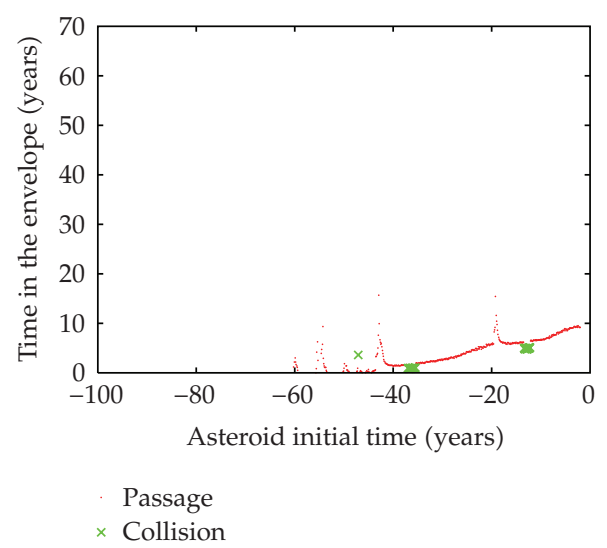

(c)

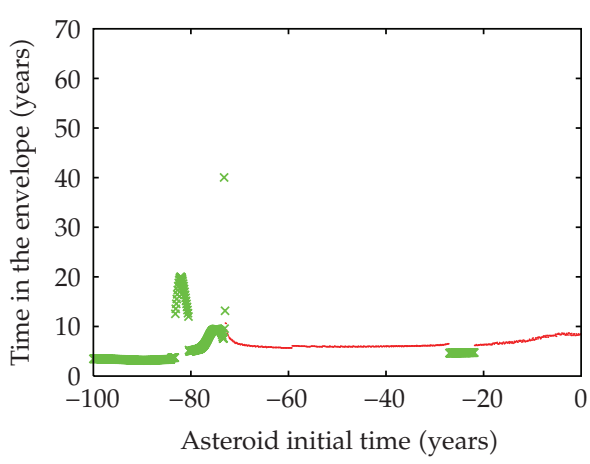

(b)

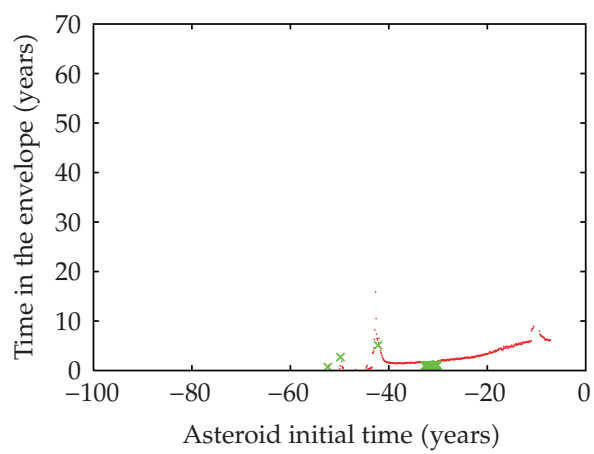

Passage

$\times$ Collision

Figure 3: Length of time that the asteroid spent inside the gas envelope for each initial time simulation. Four initial conditions for semi-major axis of $6.0 \mathrm{AU}$ : (a) $\lambda=70^{\circ}$; (b) $\lambda=110^{\circ}$; (c) $\lambda=250^{\circ}$; (d) $\lambda=290^{\circ}$.

trajectories that collide with the planet. These transition trajectories spend more time around the planet making more loops than the normal trajectories.

We studied some trajectories of Figure 4(c) and show them in Figure 5. The trajectories where condition (i) is satisfied, that is, a passage through the gas envelope, occurs when the asteroid initiated its trajectory when the gas density is close to zero. In this case the gas is so sparce that the asteroid almost does not feel it and escape (see Figure 5, trajectory d). Other possibility is when the asteroid initiated its trajectory just after the beginning of the gas density variation and the gas density is strong enough to make the asteroid to scatter in the extended atmosphere of the planet (see Figure 5, trajectory a).

Condition (ii), the collisions, mainly occurs for initial time interval $[-40,-26.1]$ years of Figure 4(c). In this time interval the gas is not so dense and allows the asteroid to enter in the envelope, but it is a trap for the asteroid which has not enough energy to escape from the gas envelope, then it spirals and collides with the planet (see Figure 5, trajectory b).

Condition (iii), integration time greater than 50 years, happens in the interval $[-26.0,-21.6]$ years of Figure 4(c). Figure 5, for trajectory c, shows a trajectory with initial time at $t=-25.0$ years. The asteroid enters the gas envelope and it is trapped by the gas. This trajectory has its semi-major axis reduced, the gas vanish and the trajectory is stabilized. 


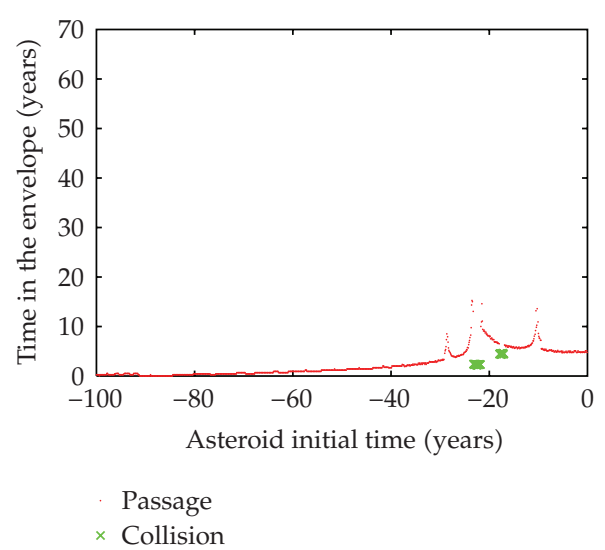

(a)

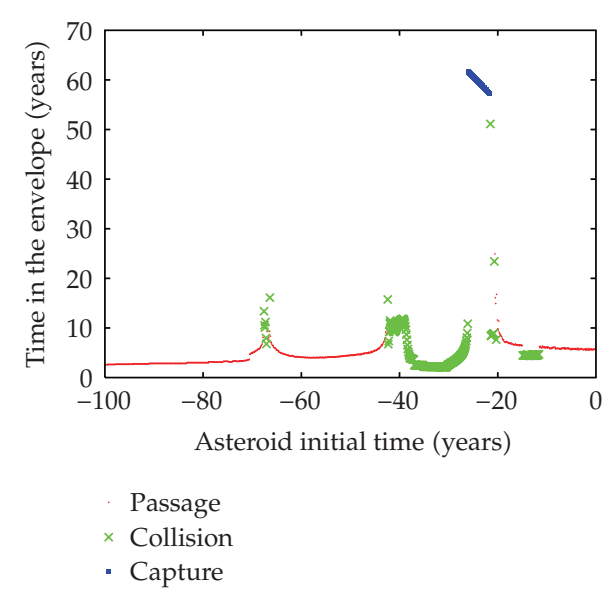

(c)

- Capture

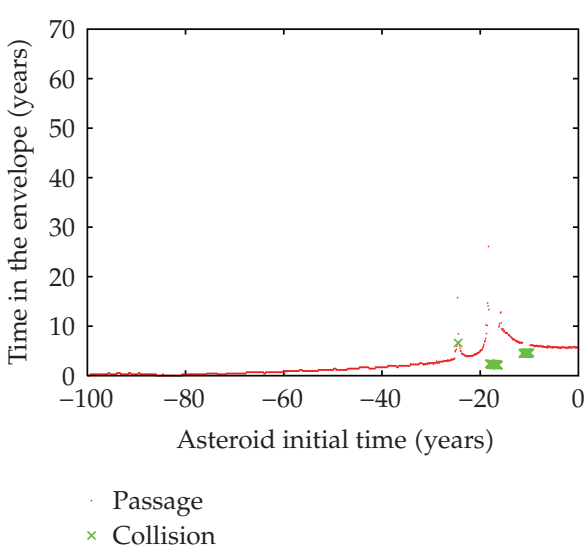

(b)

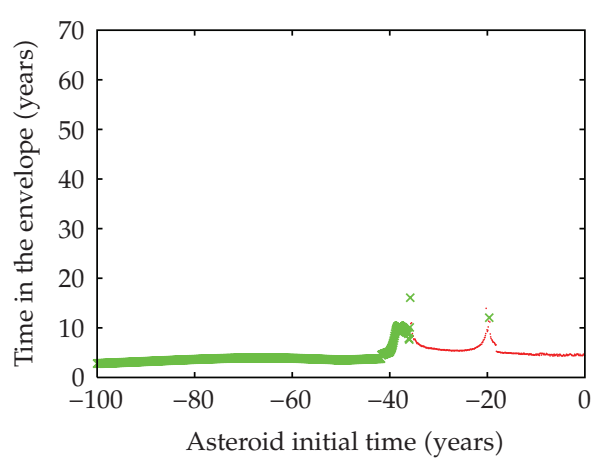

Passage

$\times$ Collision

(d)

Figure 4: Length of time that the asteroid spent inside the gas envelope for each initial time simulation. Four initial conditions for semi-major axis of $4.5 \mathrm{AU}$ : (a) $\lambda=70^{\circ}$; (b) $\lambda=110^{\circ}$; (c) $\lambda=250^{\circ}$; (d) $\lambda=290^{\circ}$.

\subsection{Captured Orbits}

In order to understand what happens with the permanently captured orbits, we computed the average of the semi-major axis and eccentricity of the captured trajectories, presented in Figure $4(\mathrm{c})$. The average was made in the time interval between 0 and 50 years, that is, after the gas had vanished.

In Figure 6 we present the behavior of the averaged semi-major axis and eccentricity after the gas vanishes. For the first trajectories, the gas is denser at the time the asteroid arrives, which make its semi-major axis to decrease more, stabilizing the orbit with a semimajor axis close to 10 Jupiter's radius. With a less dense gas at the time of arrival of the asteroid, the average value of the semi-major axis is close to 320 Jupiter's radius.

The average value of the eccentricity is also shown in Figure 6. Most of the trajectories have eccentricities close to 0.95 . The few ones which are less than 0.8 are the ones which are closer to the planet. 


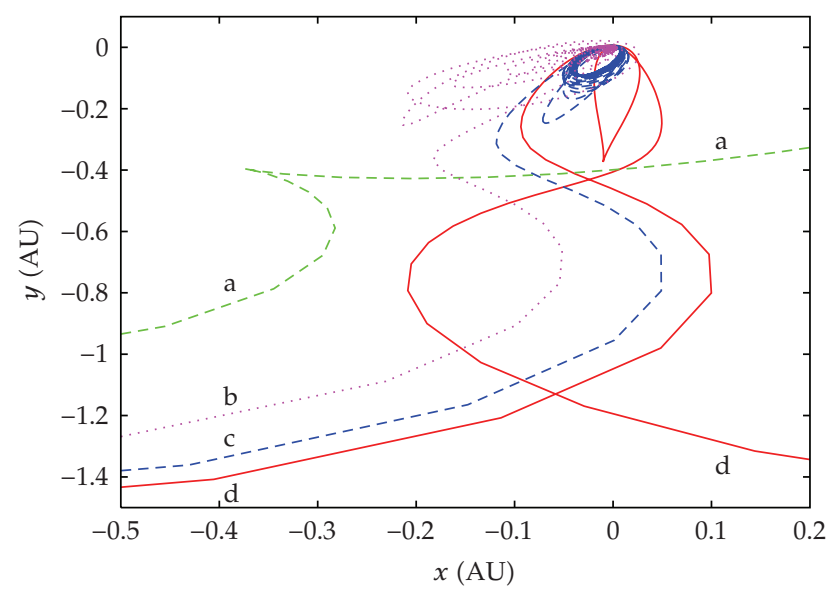

Figure 5: Four trajectories of Figure 4(c), all in a non rotating planetocentric reference frame with $x$ pointing to the Vernal point. (a) Trajectory for gas density at $t=-80$ years. The asteroid scatters in the gas and goes away. (b) Trajectory for gas density at $t=-40$ years. The asteroid enters in the gas envelope, but the gas is strong and makes the asteroid to collide with the planet. (c) Trajectory for gas density at $t=-25$ years. The asteroid enters in the gas envelope in a time that the gas is just strong enough to make the asteroid to reduce its energy and not collide with the planet. After the gas vanishes the capture is permanent. (d) Trajectory for gas density at $t=-18$ years. The asteroid has a temporary gravitational capture but the gas is too sparce and the asteroid goes away.

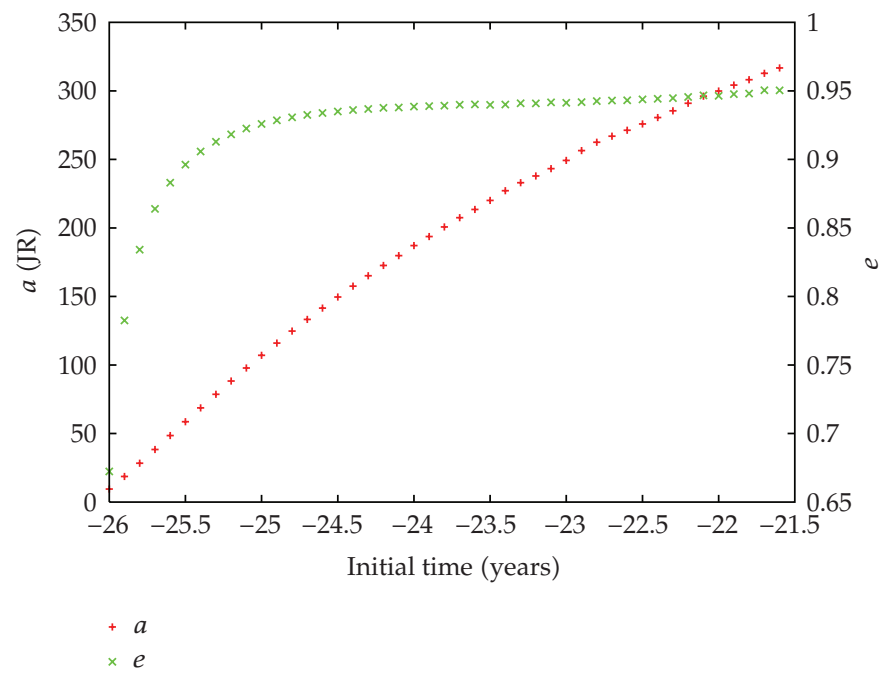

Figure 6: Averaged values of semi-major axis (left side) and eccentricity(right side) in the time interval between 0 and 50 years for the captures generated by gas drag.

We also pointed that the inclination of the asteroids during all the time after the gas was vanished for all captured trajectories were retrograde, that is, the inclinations never changes from $i=180^{\circ}$ with respect to Jupiter.

This process of capture is not suitable for the prograde capture. In the work of Ćuk and Burns [14] they show that the time needed to capture a Himalia satellite type is between $10^{4}$ to $10^{6}$ years. The process described here is rapid and the density of the gas envelope is 
not enough to make a asteroid to lose orbital energy and turn it into a permanent prograde satellite.

If we compare this and the work of Ćuk and Burns [14], using the results of Pollack et al. [15], the capture of the prograde satellites might have occurred in the second phase of planetary formation, while the capture of the retrograde satellites might have occurred only in the third stage. In the work of Pollack et al. [15] they estimate a time between $10^{6}$ years and $8 \times 10^{6}$ years in the second phase. Thus, the model of capture with gas drag show us that the capture of prograde satellites are much more probable than the retrograde ones. This contradicts the observations. There are more retrograde satellites observed than prograde ones. Therefore, the origin of the prograde and retrograde irregular satellites cannot be attributed to the gas drag capture mechanism alone.

\section{Conclusions}

We found a region of initial conditions, close to Jupiter, which produces temporary gravitational capture around Jupiter. We took eight sets of initial conditions from this region and varied the initial time for the integration in a dynamics which includes a gas envelope with decreasing density along the time to a moment that it vanishes. These simulations gave us information about what might be the outcomes of the evolution of such system. The possible outcomes are: the scatter of the asteroid, the temporary gravitational capture, the collision with Jupiter, and the permanent capture. Seven out of eight sets of initial conditions did not give effective capture. Only one set presented a few permanent captures due to the gas drag.

We have shown that the permanent capture of an asteroid in retrograde orbit around Jupiter due to a vanishing gas envelope is possible. We have the knowledge that this happens with low probability, but considering the supply of planetesimals available in the early time of the solar system formation, this kind of event could have happened. Although comparing with the probability of prograde capture it is less probable. The observations show more retrograde satellites than prograde ones. This is a problem for the gas drag capture mechanism as a whole.

It is necessary to look more regions of the space of semi-major axis, eccentricity, and inclination in order to have enough information about the gravitational capture with gas drag and also the time for the gas envelope to vanish needs to be tested. In order to implement this it is necessary to use some Monte Carlo method due to the huge range of free parameters.

The captures studied in this work, which were shown in Section 3.2, are close to Jupiter compared with the real irregular satellites. Some could cross the Galilean satellites orbits and collide with them. It is necessary to improve the gas envelope decaying mechanism to obtain more information of the process, but in this work we tried to proof this concept and further work is under development.

\section{Acknowledgments}

This work was funded by FAPESP and CNPq. These supports are gratefully acknowledged.

\section{References}

[1] J. B. Pollack, J. A. Burns, and M. E. Tauber, "Gas drag in primordial circumplanetary envelopes: a mechanism for satellite capture," Icarus, vol. 37, no. 3, pp. 587-611, 1979. 
[2] B. J. Gladman, P. D. Nicholson, J. A. Burns, et al., "Discovery of two distant irregular moons of Uranus," Nature, vol. 392, no. 6679, pp. 897-899, 1998.

[3] D. Jewitt and N. Haghighipour, "Irregular satellites of the planets: products of capture in the early solar system," Annual Review of Astronomy and Astrophysics, vol. 45, pp. 261-295, 2007.

[4] K. Tanikawa, "Impossibility of the capture of retrograde satellites in the restricted three-body problem," Celestial Mechanics, vol. 29, no. 4, pp. 367-402, 1983.

[5] E. Vieira Neto and O. C. Winter, "Time analysis for temporary gravitational capture: satellites of Uranus," Astronomical Journal, vol. 122, no. 1, pp. 440-448, 2001.

[6] D. S. de Oliveira, O. C. Winter, E. Vieira Neto, and G. de Felipe, "Irregular satellites of Jupiter: a study of the capture direction," Earth, Moon and Planets, vol. 100, no. 3-4, pp. 233-239, 2007.

[7] T. A. Heppenheimer and C. Porco, "New contributions to the problem of capture," Icarus, vol. 30, no. 2, pp. 385-401, 1977.

[8] E. Vieira Neto, O. C. Winter, and T. Yokoyama, "The effect of Jupiter's mass growth on satellite capture Retrograde case," Astronomy and Astrophysics, vol. 414, no. 2, pp. 727-734, 2004.

[9] E. Vieira Neto, O. C. Winter, and T. Yokoyama, "Effect of Jupiter's mass growth on satellite capture the prograde case," Astronomy and Astrophysics, vol. 452, no. 3, pp. 1091-1097, 2006.

[10] G. Colombo and F. A. Franklin, "On the formation of the outer satellite groups of Jupiter," Icarus, vol. 15, no. 2, pp. 186-189, 1971.

[11] C. B. Agnor and D. P. Hamilton, “Neptune's capture of its moon Triton in a binary-planet gravitational encounter," Nature, vol. 441, no. 7090, pp. 192-194, 2006.

[12] H. S. Gaspar, O. C. Winter, and E. Vieira Neto, "Irregular satellites of Jupiter: capturecongurations of binary asteroids," submitted to Monthly Notices of the Royal Astronomical Society.

[13] J. I. Lunine and D. J. Stevenson, "Formation of the Galiean satellites in a gaseous nebula," Icarus, vol. 52, pp. 14-39, 1982.

[14] M. Ćuk and J. A. Burns, “Gas-drag-assisted capture of Himaila's family,” Icarus, vol. 167, pp. 369-381, 2004.

[15] J. B. Pollack, O. Hubickyj, P. Bodenheimer, J. J. Lissauer, M. Podolak, and Y. Greenzweig, “Formation of the giant planets by concurrent accreation of solid and gas," Icarus, vol. 124, pp. 62-85, 1996.

[16] E. Vieira Neto, O. C. Winter, and C. Melo, "The use of the two-body energy to study problems of escape/capture," in Dynamics of Populations of Planetary Systems, Z. Knezevic and A. Milani, Eds., Proceedings IAU Colloquium, no. 197, pp. 439-444, Cambridge University Press, Cambridge, UK, 2005.

[17] E. Everhart, "An efficient integrator that uses Gauss-Radau spacings," in Dynamics of Comets: Their Origin and Evolution, A. Carusi and G. B. Valsecchi, Eds., vol. 115 of Proceedings of IAU Colloquium, no. 83, pp. 185-202, Astrophysics and Space Science Library, Dordrecht, The Netherlands, 1985.

[18] C. D. Murray and S. F. Dermott, Solar System Dynamics, Cambridge University Press, Cambridge, UK, 1999.

[19] J. Wisdom, "The resonance overlap criterion and the onset of stochastic behavior in the restricted three-body problem," Astronomical Journal, vol. 85, pp. 1122-1133, 1980.

[20] M. Podolak, W. B. Hubbard, and J. B. Pollack, "Gaseous acreation and the formation of giant planets," in Protostars and Planets III, E. H. Levy and J. I. Lunini, Eds., pp. 1109-1147, University of Arizona Press, Tucson, Ariz, USA, 1993.

[21] I. Adashi, C. Hayashi, and K. Nakazawa, "The gas drag effect on the elliptic motion of a solid body in the primordial nebula," Progress in Theorical Physics, vol. 56, no. 3, pp. 1756-1771, 1976.

[22] S. J. Weidenschilling, "Planetesimals from Stardust," in From Stardust to Planetesimals, Y. J. Pendleton and A. G. G. M. Tielens, Eds., vol. 122 of ASP Conference Series, p. 281, Astronomical Society of the Pacific, Santa Clara, Calif, USA, 1997. 


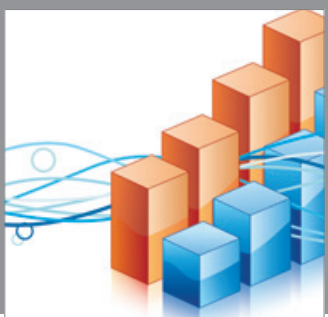

Advances in

Operations Research

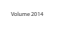

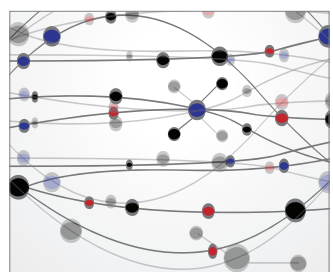

\section{The Scientific} World Journal
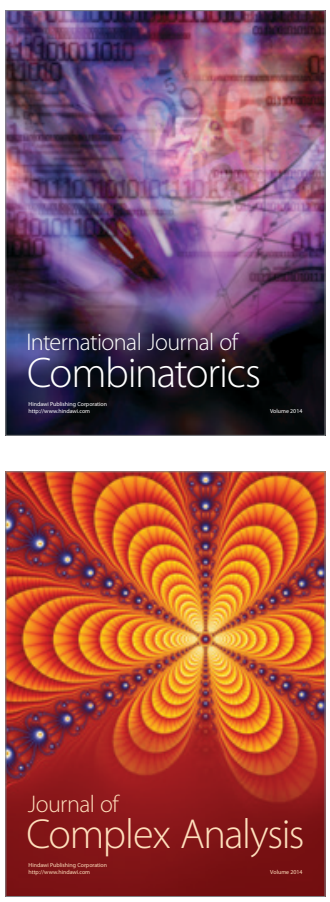

International Journal of

Mathematics and

Mathematical

Sciences
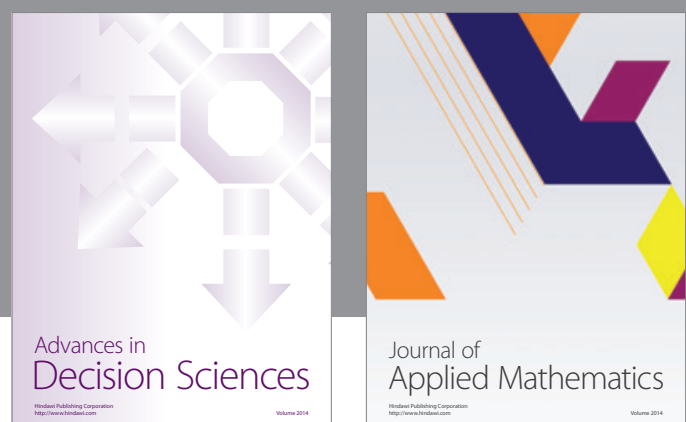

Journal of

Applied Mathematics
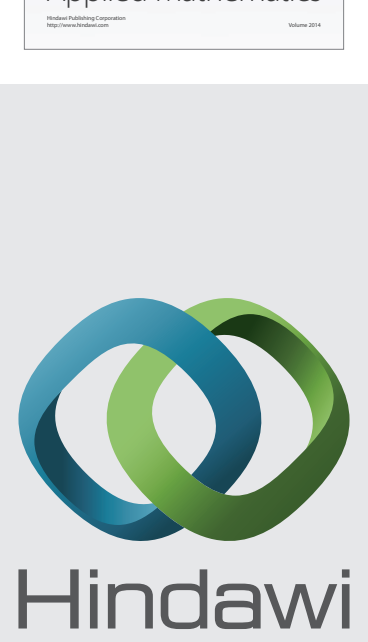

Submit your manuscripts at http://www.hindawi.com
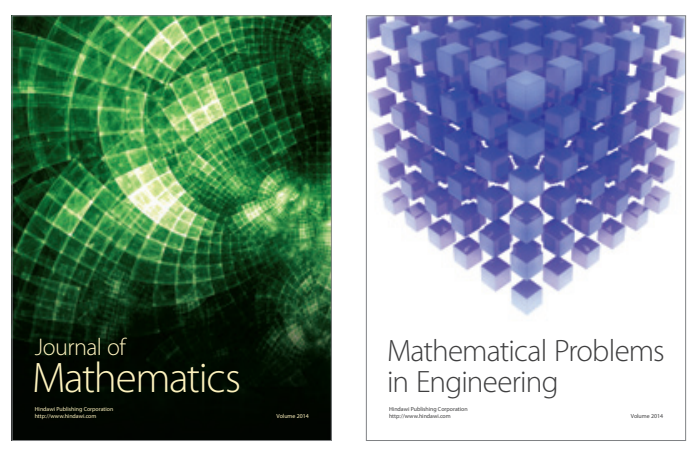

Mathematical Problems in Engineering
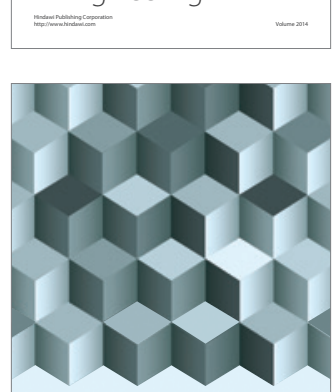

Journal of

Function Spaces
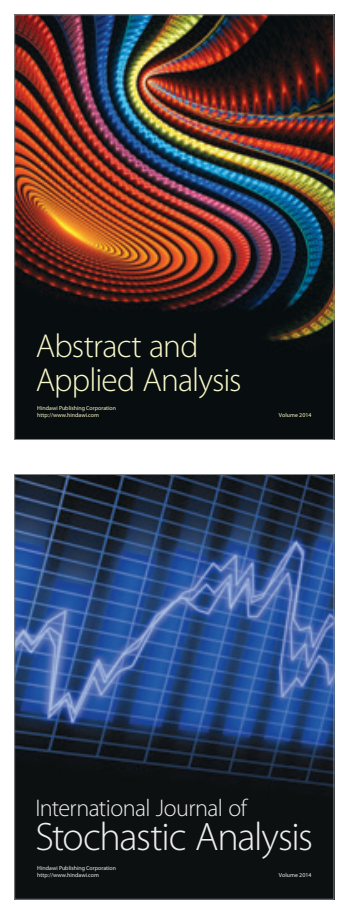

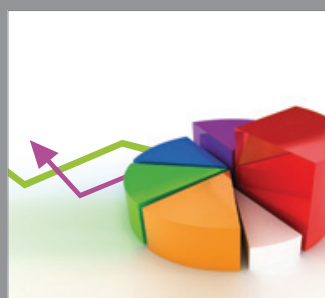

ournal of

Probability and Statistics

Promensencen
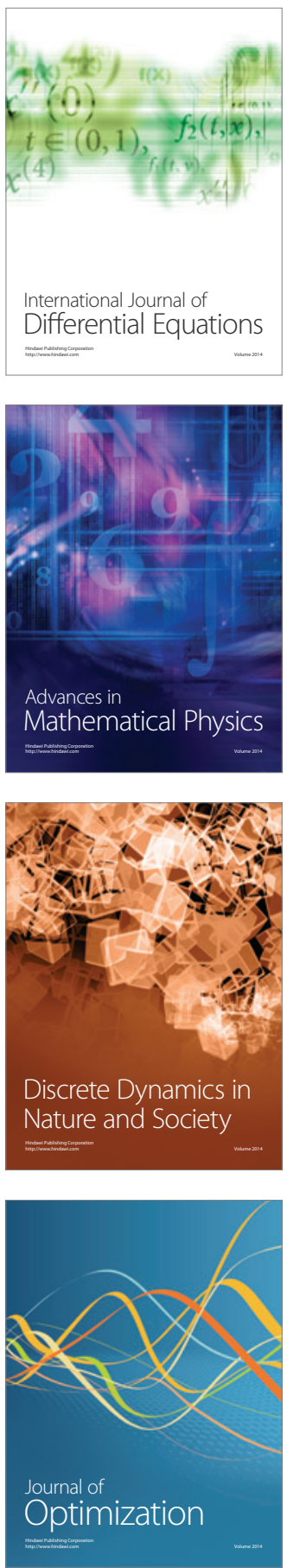